PROCEEDINGS OF THE

AMERICAN MATHEMATICAL SOCIETY

Volume 131, Number 11, Pages 3527-3534

S 0002-9939(03)07035-7

Article electronically published on May 7, 2003

\title{
AN ALGEBRAIC FORMULATION OF THURSTON'S COMBINATORIAL EQUIVALENCE
}

\author{
KEVIN M. PILGRIM
}

(Communicated by Michael Handel)

\begin{abstract}
Let $f: S^{2} \rightarrow S^{2}$ be an orientation-preserving branched covering for which the set $P_{f}$ of strict forward orbits of critical points is finite and let $G=\pi_{1}\left(S^{2}-f^{-1} P_{f}\right)$. To $f$ we associate an injective endomorphism $\varphi_{f}$ of the free group $G$, well-defined up to postcomposition with inner automorphisms. We show that two such maps $f, g$ are combinatorially equivalent (in the sense introduced by Thurston for the characterization of rational functions as dynamical systems) if and only if $\varphi_{f}, \varphi_{g}$ are conjugate by an element of $\operatorname{Out}(G)$ which is induced by an orientation-preserving homeomorphism.
\end{abstract}

\section{INTRODUCTION}

We trace an explicit path from the combinatorial theory of rational maps as holomorphic dynamical systems to the theory of injective endomorphisms of free groups as algebraic dynamical systems.

Following [DH], let $f: S^{2} \rightarrow S^{2}$ be an orientation-preserving branched covering map of degree $d \geq 2$. The postcritical set is $P_{f}=\bigcup_{n>0} f^{\circ n}\left(C_{f}\right)$ where $C_{f}$ is the set of critical points of $f$. We say $f$ is postcritically finite, or a Thurston map, if $P_{f}$ is finite. Two Thurston maps $f, g$ are combinatorially equivalent (we henceforth drop the adjective, combinatorial) if there are orientation-preserving homeomorphisms $\phi_{0}, \phi_{1}:\left(S^{2}, P_{f}\right) \rightarrow\left(S^{2}, P_{g}\right)$ such that $\phi_{0}$ is isotopic to $\phi_{1}$ relative to $P_{f}$ and $\phi_{0} \circ f=g \circ \phi_{1}$.

In the mid-1980's W. Thurston (i) showed that apart from a well-understood set of exceptions, a postcritically finite rational map, as a holomorphic dynamical system, is determined up to Möbius conjugacy by its equivalence class; and (ii) characterized completely (although rather implicitly) the set of Thurston maps which are equivalent to rational maps; see $\S 3$ for precise statements. These results have been used to give, for example, combinatorial descriptions of various parameter spaces and combination procedures for rational maps, viewed as dynamical systems.

In practice, it can be difficult to determine when two explicitly given Thurston maps are equivalent (see $\S 4$ ). Since equivalence is defined in terms of topological data up to isotopy, it is natural to seek an algebraic formulation of this notion.

Received by the editors June 20, 2002.

2000 Mathematics Subject Classification. Primary 37F20; Secondary 20F28, 20F36, 20 E05.

Key words and phrases. Postcritically finite, endomorphism of free group.

This research was supported by Indiana University, Bloomington. 
In this work, we show that a Thurston map $f$ induces a monomorphism $\varphi_{f}: G \rightarrow$ $G$ which is well-defined up to postcomposition by inner automorphisms, where $G=$ $\pi_{1}\left(S^{2}-f^{-1} P_{f}\right)$ is a free group of finite rank. We also show that $f$ is equivalent to $g$ if and only if $\varphi_{f}$ and $\varphi_{g}$ are conjugate via an element $\phi_{1 *}$ of $\operatorname{Out}(G)$ which is induced from an orientation-preserving homeomorphism $\phi_{1}$; see $\S 2$. Thus, equivalence of Thurston maps can be characterized algebraically.

In contrast to to the present work, A. Kameyama in $\mathrm{Kam}$ associates to a Thurston map $f$ a certain homomorphism $f_{\dagger}: \pi_{1}\left(S^{2}-P_{f}\right) \rightarrow \pi_{1}\left(S^{2}-P_{f}\right) \rtimes S_{d}$ between different groups; here $S_{d}$ is the symmetric group on $d=\operatorname{deg}(f)$ letters. There, the main result gives necessary and sufficient conditions in terms of $f_{\dagger}, g_{\dagger}$ for two Thurston maps $f, g$ to be equivalent. From this an alternative, somewhat more complicated, algebraic criterion for equivalence follows easily.

In $\S 2$, we formulate precisely and prove our main result, Theorem 2.4. Injections of free groups to themselves have been objects of recent study [Kap], [DV]. In $\S 3$ we state precisely Thurston's characterization theorem, discuss possible translations of this characterization into group-theoretic language, and list some elementary properties of $\varphi_{f}$ from a combinatorial group theory point of view. We conclude in $\S 4$ with a discussion of J. Hubbard's "twisted rabbit" problem.

I thank G. Baumslag, B. Farb, W. Floyd, M. Handel, and I. Kapovich for useful conversations and references.

\section{Statements AND PROOFS}

2.1. Notation. Here $\mathbb{C}$ is the complex plane, $\widehat{\mathbb{C}}$ is the Riemann sphere, and $f^{\circ l}$ is the $l$-fold composition of a function $f$ with itself. Fix distinct points $a_{1}, \ldots, a_{p}$, $a_{p+1}, \ldots, a_{q}$ in $\mathbb{C}$. Let $a_{0}=\infty, A_{0}=\left\{a_{0}, \ldots, a_{p}\right\}, A_{1}=A_{0} \cup\left\{a_{p+1}, \ldots, a_{q}\right\}$, and $X_{k}=\widehat{\mathbb{C}}-A_{k}, k=0,1$.

Fix $b \in X_{1}$. For each $i=1, \ldots, q$ let $\alpha_{i}$ denote a simple, counterclockwise oriented simple closed curve based at $b$ winding once around the puncture $a_{i}$, such that $\alpha_{i} \cap \alpha_{j}=\{b\}$ for all $i \neq j$. Note that $\bigcup_{1}^{p} \alpha_{i}$ and $\bigcup_{1}^{q} \alpha_{i}$ are homotopy equivalent to $X_{0}$ and $X_{1}$, respectively. Let $x_{i}=\left[\alpha_{i}\right] \in \pi_{1}\left(X_{1}, b\right)$. Let

$$
G_{1}=\pi_{1}\left(X_{1}, b\right) \simeq\left\langle x_{1}, \ldots, x_{p}, x_{p+1}, \ldots, x_{q}\right\rangle
$$

and let

$$
G_{0}=\pi_{1}\left(X_{0}, b\right) \simeq\left\langle x_{1}, \ldots, x_{p}\right\rangle .
$$

It will sometimes be convenient not to regard $G_{0}$ as a subgroup of $G_{1}$. More precisely, the inclusion $\iota: X_{1} \hookrightarrow X_{0}$ induces a surjection $\iota_{*}: G_{1} \rightarrow G_{0}$ with kernel $N_{1}$, the normal subgroup of $G_{1}$ generated by $\left\{x_{j}\right\}_{j=p+1}^{q}$. The exact sequence

$$
1 \rightarrow N_{1} \rightarrow G_{1} \stackrel{\iota *}{\rightarrow} G_{0} \rightarrow 1
$$

splits. We choose and fix the section $\sigma_{*}: G_{0} \rightarrow G_{1}$ of $\iota_{*}$ given by $G_{0} \ni x_{i} \mapsto$ $x_{i} \in G_{1}$. Thus $\sigma_{*}$ is induced by the inclusion $\sigma: \bigcup_{1}^{p} \alpha_{i} \hookrightarrow \bigcup_{1}^{q} \alpha_{i}$ which sends $\alpha_{i} \mapsto \alpha_{i}, i=1, \ldots, p$.

2.2. Branched covers. An orientation-preserving, continuous map $f: \widehat{\mathbb{C}} \rightarrow \widehat{\mathbb{C}}$ is a branched covering if can be written in the form $z \mapsto z^{k}$ in local complex coordinates. If $k \geq 2$ the point corresponding to the origin is called a critical point of $f$ of local degree $k$.

It is often convenient to keep track of sets which may be larger than the postcritical set. Fix an integer $d \geq 2$ and sets $A_{0}, A_{1}$ as in the previous section. Let 
$\mathcal{B}$ denote the set of branched covers $f: \widehat{\mathbb{C}} \rightarrow \widehat{\mathbb{C}}$ such that the critical points of $f$ lie in $A_{1}, \operatorname{deg}(f)=d$, and $A_{1}=f^{-1}\left(A_{0}\right)$. Note that $f \in \mathcal{B}$ implies $P_{f} \subset A_{0}$. Any Thurston map $g$ of degree $d$ having $\# P_{g} \leq \# A_{0}$ is topologically conjugate by an orientation-preserving homeomorphism to an element of $\mathcal{B}$.

Definition 2.1. Maps $f, g \in \mathcal{B}$ are called equivalent if there exist orientationpreserving homeomorphisms $\phi_{0}, \phi_{1}: \widehat{\mathbb{C}} \rightarrow \widehat{\mathbb{C}}$ such that (i) $\phi_{k}\left(A_{k}\right)=A_{k}, k=0,1$; (ii) the diagram

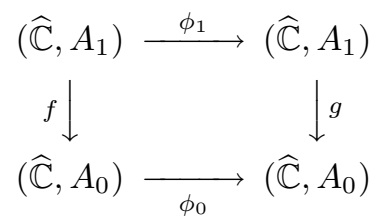

commutes; and (iii) $\phi_{0}$ and $\phi_{1}$ are isotopic through homeomorphisms agreeing on $A_{0}$.

Definition 2.2. For $f \in \mathcal{B}$, let $\varphi_{f}: G_{1} \rightarrow G_{1}$ be given by $\varphi_{f}=\sigma_{*} \circ f_{*}$, where $f_{*}: G_{1} \rightarrow G_{0}$ is the map induced by the unramified covering $f \mid X_{1}: X_{1} \rightarrow X_{0}$ on fundamental groups.

Remark 2.3. (i) If $G$ is a group and $g \in G$, the map $x \mapsto g x g^{-1}$ defines an inner automorphism of $G$. The group of inner automorphisms is a normal subgroup of the full group of automorphisms, and the corresponding quotient is called the group of outer automorphisms, denoted $\operatorname{Out}(G)$. Since $f$ need not send the basepoint $b$ to itself, the induced map $f_{*}$ is defined only up to postcomposition with an inner automorphism of $G_{0}$. Hence $\varphi_{f}$ is defined only up to postcomposition with an inner automorphism of $G_{1}$. (ii) Since $f_{*}$ and $\sigma_{*}$ are injective, so is $\varphi_{f}$. (iii) $\varphi_{f}$ sends a word in $x_{1}, \ldots, x_{q}$ to a word in $x_{1}, \ldots, x_{p}$. Thus, $\varphi_{f}\left(G_{1}\right) \subset\left\langle x_{1}, \ldots, x_{p}\right\rangle$.

The main result of this paper is

Theorem 2.4. Suppose $f, g \in \mathcal{B}$. Then $f$ and $g$ are equivalent, via a pair of orientation-preserving homeomorphisms $\left(\phi_{0}, \phi_{1}\right)$, if and only if $\varphi_{g}=\phi_{1 *} \circ \varphi_{f} \circ \phi_{1 *}^{-1}$ up to inner automorphisms.

Since there are purely algebraic conditions for an automorphism $G_{1} \rightarrow G_{1}$ to be induced by a homeomorphism $\phi_{1}: X_{1} \rightarrow X_{1}$ (see [ZVC], Thm. 5.7.1), the preceding theorem represents a purely algebraic formulation of Thurston equivalence.

Lemma 2.5. Suppose $\phi_{k}: \widehat{\mathbb{C}} \rightarrow \widehat{\mathbb{C}}$ are orientation-preserving homeomorphisms for which $\phi_{k}\left(A_{k}\right)=A_{k}, k=0,1$. Let $\phi_{k *}: G_{k} \rightarrow G_{k}$ be the maps on fundamental groups induced by restricting $\phi_{k}$ to $X_{k}, k=0,1$. Then

(1) $\phi_{1 *}\left(N_{1}\right) \subset N_{1}$, and therefore $\phi_{1 *}$ induces a map $\bar{\phi}_{1 *}: G_{0} \rightarrow G_{0}$;

(2) $\phi_{0}$ is isotopic to $\phi_{1}$ relative to $A_{0}$ if and only if $\bar{\phi}_{1 *}=\phi_{0 *}$ up to composition with inner automorphisms;

(3) if $\phi_{0}$ is isotopic to $\phi_{1}$ relative to $A_{0}$, then $\phi_{1 *} \circ \sigma_{*}=\sigma_{*} \circ \phi_{0 *}$.

Proof. 1. Let $\alpha:\left(S^{1}, 1\right) \rightarrow\left(X_{1}, b\right)$ represent an element of $N_{1}$. Then there is a homotopy in $X_{0}$ from $\alpha$ to the constant map at $b$. Since $\phi_{1}$ sends $A_{k}$ to itself, $k=0,1$, it sends $X_{0}$ to itself. Hence composition of the homotopy with $\phi_{1}$ yields a homotopy in $X_{0}$ of $\phi_{1} \circ \alpha$ with the constant map at $\phi_{1}(b)$. 2. Necessity is clear. Sufficiency follows from the fact that the isotopy class of a surface homeomorphism 
is determined by its action on the fundamental group. 3. This follows immediately from conclusion (2) and the definitions.

Proof of Theorem 2.4. Suppose that $f$ is equivalent to $g$ via $\left(\phi_{0}, \phi_{1}\right)$. Then $g=$ $\phi_{0} \circ f \circ \phi_{1}^{-1}$. Passing to induced maps on fundamental groups, we have that up to postcomposition with inner automorphisms, $\varphi_{g}=\sigma_{*} \circ g_{*}=\sigma_{*} \circ \phi_{0 *} \circ f_{*} \circ \phi_{1 *}^{-1}$. By conclusion (3) of the lemma, this in turn is $\phi_{1 *} \circ \sigma_{*} \circ f_{*} \circ \phi_{1 *}^{-1}=\phi_{1 *} \circ \varphi_{f} \circ \phi_{1 *}^{-1}$.

Conversely, suppose that up to inner automorphism one has $\varphi_{g}=\phi_{1 *} \circ \varphi_{f} \circ \phi_{1 *}^{-1}$ for some homeomorphism $\phi_{1}:\left(\widehat{\mathbb{C}}, A_{1}\right) \rightarrow\left(\widehat{\mathbb{C}}, A_{1}\right)$ for which $\phi_{1}: A_{0} \rightarrow A_{0}$. Then $\sigma_{*} \circ g_{*} \circ \phi_{1 *}=\phi_{1 *} \circ \sigma_{*} \circ f_{*}$.

Composing this equality on the left with $\iota_{*}$ yields (using the definitions and the fact that $\sigma_{*}$ is a section of $\iota_{*}$ ) the identity $g_{*} \circ \phi_{1 *}=\bar{\phi}_{1 *} \circ f_{*}$.

Next, let $\phi_{0}=\phi_{1}$. Then $\phi_{0 *}=\bar{\phi}_{1 *}$ and we may write the previous identity as

$$
g_{*} \circ \phi_{1 *}=\phi_{0 *} \circ f_{*} \text {. }
$$

The conclusion of the previous paragraph implies that $\phi_{0}$ lifts under $f, g$ to a map $\widetilde{\phi}_{0}: \widehat{\mathbb{C}} \rightarrow \widehat{\mathbb{C}}$ sending $A_{k}$ to $A_{k}, k=0,1$, such that $g \circ \widetilde{\phi}_{0}=\phi_{0} \circ f$. On the level of fundamental groups, up to inner automorphisms

$$
g_{*} \circ \widetilde{\phi}_{0 *}=\phi_{0 *} \circ f_{*} .
$$

The right-hand sides of (2.1) and (2.2) are the same, and $g_{*}$ is injective, hence up to inner automorphisms $\phi_{1 *}=\widetilde{\phi}_{0 *}$. The homeomorphisms $\widetilde{\phi}_{0}$ and $\phi_{1}=\phi_{0}$ are therefore isotopic relative to $A_{1}$. Thus $f$ and $g$ are equivalent via the pair $\left(\phi_{0}, \widetilde{\phi}_{0}\right)$ where $\left(\widetilde{\phi}_{0}\right)_{*}=\phi_{1 *}$.

\section{Properties of $\varphi_{f}$}

It is natural to seek a translation of Thurston's characterization of rational functions into properties of the map $\varphi_{f}$. For definitions, see $[\mathrm{DH}]$.

Theorem 3.1 (Thurston's characterization and rigidity theorem). A Thurston map $f$ with hyperbolic orbifold $\mathcal{O}_{f}$ is equivalent to a rational function if and only if for any $f$-stable multicurve $\Gamma$ we have $\lambda(f, \Gamma)<1$. In that case, the rational function is unique up to conjugation by an automorphism of the Riemann sphere.

If $\mathcal{O}_{f}$ is hyperbolic and $\Gamma$ is an $f$-stable multicurve with $\lambda(f, \Gamma) \geq 1$, we will call $\Gamma$ a Thurston obstruction. Although obstructions are far from unique, it is shown in [Pil2] that in fact the iteration on Teichmüller space employed in the proof of this theorem picks out a canonical one, if any obstruction exists. A combinatorial characterization of this canonical obstruction, however, is not yet known.

A translation of this criterion into properties of the maps $\varphi_{f}, \iota_{*}, \sigma_{*}$ seems likely to be cumbersome in general.

We content ourselves here with a discussion of a simple kind of obstruction, namely when $f$ has a Levy cycle, i.e. a cycle of pairwise disjoint, essential, nonperipheral, simple, closed curves mapping by degree one (peripheral means freely homotopic to a loop surrounding only one puncture). An obstructed degree two Thurston map (or, more generally, one with exactly two critical points) always has a Levy cycle (Tan], Cor. 3.2), although this fails in higher degrees $\underline{\mathrm{ST}}$. More generally, a rational map with hyperbolic orbifold cannot have a cycle of non-simple closed curves mapping by degree one, or still more generally, a cycle of non-simple, possibly intersecting closed curves mapping by degree one. To see this, note that by 
the Schwarz-Pick lemma, the restriction $\left.f\right|_{X_{1}}: X_{1} \rightarrow X_{0}$ is expanding with respect to the hyperbolic metric on $X_{0}$, which contains $X_{1}$ since $A_{1}$ strictly contains $A_{0}$. The inverse branches are therefore contracting. Hence if $\gamma_{1}, \gamma_{2}$ are closed nonperipheral curves for which $\left.f\right|_{\gamma_{1}}: \gamma_{1} \rightarrow \gamma_{2}$ by degree one, then $l\left(\gamma_{1}\right)<l\left(\gamma_{2}\right)$, where $l(\gamma)$ denotes the length of the unique hyperbolic geodesic on $X_{0}$ homotopic to $\gamma$.

Theorem 3.2. A degree two Thurston map $f$ with hyperbolic orbifold is obstructed if and only if there is a sequence $\left\{g_{i}\right\}_{i=0}^{l-1} \subset G_{1}$ such that for $0 \leq i \leq l-1$,

(1) (nonperipheral) $\iota_{*}\left(g_{i}\right)$ is nontrivial and is not conjugate in $G_{0}$ to any $x_{j}$, $1 \leq j \leq p$ or to $\prod_{j=1}^{p} x_{j}$ (the latter product is represented by a peripheral curve about the point at infinity);

(2) (degree one) $\iota_{*}\left(g_{i}\right)$ is primitive in $G_{0}$, i.e. is not a power of another element;

(3) (periodic) $\iota_{*}\left(\varphi_{f}\left(g_{i}\right)\right)=\iota_{*}\left(g_{i+1}\right)$ up to conjugacy in $G_{0}$ (subscripts read modulo $n)$.

Proof. Suppose such an $f$ is obstructed. Then by Tan it has a Levy cycle and necessity follows easily. To prove the sufficiency, represent each $g_{i}$ by an oriented curve $\gamma_{i} \subset X_{1}$. Condition (1) says that for each $i$, the curve $\iota\left(\gamma_{i}\right)$ is essential and nonperipheral in $X_{0}$; condition (3) is equivalent to $f_{*}\left(g_{i}\right)=\iota_{*}\left(g_{i+1}\right)$, implying that $\iota\left(\gamma_{i+1}\right)$ lifts under $f$ to $\gamma_{i}$; condition (2) implies that the degree of $f$ restricted to this lift is one, as required.

Remark 3.3. The case when $p=2$ is interesting as well (see e.g. Pill for connections to geometric Galois theory), as then there are no multicurves, hence no obstructions and so any such Thurston map is equivalent to a rational map. Therefore any general translation of Thurston's criterion into group theory must accommodate this fact.

Fixed-point sets of endomorphisms of free groups were studied in [DV].

Corollary 3.4. If $f$ is equivalent to a rational map, and if every cycle in $P_{f}$ contains a critical point (i.e. the rational map is hyperbolic), then for all $l>0$, the set $\operatorname{Fix}\left(\varphi_{f}^{o l}\right)=\left\{x \in G_{1}: \varphi_{f}^{o l}(x)=x\right\}$ is the trivial group $\{1\}$.

Proof. A nontrivial fixed element yields, by the constructions in the proof of Theorem 3.2 above, a cycle (up to homotopy) of essential, possibly peripheral closed curves, each mapping by degree one. The elements of a cycle of peripheral curves cannot all map by degree one, by the assumption on $P_{f}$ (compare the proof of Theorem 3.5 below). So, a nontrivial element of $\operatorname{Fix}\left(\varphi_{f}^{o l}\right)$ yields an obstruction.

Let $G$ be the free group on a finite set of symbols $Y$ and $\varphi: G \rightarrow G$ an injective homomorphism. The mapping torus of $\varphi$ is defined by $M_{\varphi}=\langle t, Y| t^{-1} y t=$ $\varphi(y) \quad \forall y \in Y\rangle$. Its isomorphism type is unchanged if $\varphi$ is replaced by either $g \varphi g^{-1}, g \in G$, or by $\psi^{-1} \varphi \psi$ where $\psi \in \operatorname{Aut}(G)$. By results of M. Feighn and M. Handel $\left[\mathrm{FH}\right.$ and R. Geoghegan et al. GMSW, respectively, $M_{\varphi}$ is coherent (all finitely generated subgroups are finitely presented) and Hopfian (not isomorphic to any proper quotient). The question of when $M_{\varphi}$ is word-hyperbolic is addressed in Kap. There, it is shown that if there exist "periodic conjugacy classes", i.e. nontrivial $g \in G$ for which $\varphi^{o l}(g)$ is conjugate to $g^{m}$ for some integers $l, m>0$, then $M_{\varphi}$ is not word-hyperbolic.

Theorem 3.5. Let $\varphi: G_{1} \rightarrow G_{1}$ be an injection. If $\varphi=\varphi_{f}$ for some Thurston map $f$, then $M_{\varphi}$ is not word-hyperbolic. 


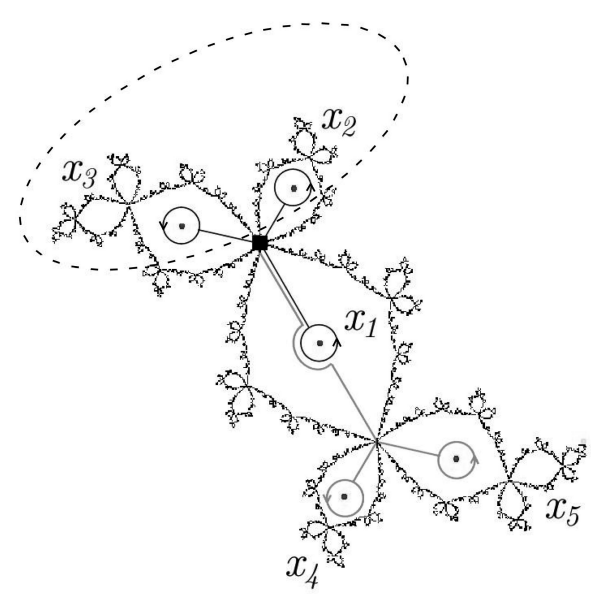

Figure 1. Twisting the Douady rabbit

Proof. Let $f$ be a Thurston map. Since the set $A_{1}$ is finite and forward-invariant, it contains a periodic cycle, say of period $l$. If $m$ is the local degree of $f^{\circ l}$ computed at any point $a$ in the cycle, then a sufficiently small oriented peripheral loop $\gamma$ surrounding $a$ will have the property that $f^{\circ l}(\gamma)$ is freely homotopic to the oriented curve $\gamma$ traversed $m>0$ times. Thus $\varphi_{f}^{o l}([\gamma])$ is conjugate to $[\gamma]^{m}$, where $1 \neq[\gamma] \in$ $G_{1}$.

Remark 3.6. An injective endomorphism $\varphi$ of a free group $G$ is called an immersion if, for every $y, y^{\prime} \in Y \cup Y^{-1}$ for which $y^{-1} \neq y^{\prime}$, the word $\varphi(y) \varphi\left(y^{\prime}\right)$ is freely reduced as written, i.e. no cancellation occurs. The notion was introduced by J. Stallings Sta and has since played an important role in the study of endomorphisms of free groups. Immersions are plentiful and are more tractable to analyze. For immersions, I. Kapovich proves that $M_{\varphi}$ is hyperbolic if and only there no periodic conjugacy classes. This condition is conjectured to be necessary and sufficient for arbitrary $\varphi$; by results of $\mathrm{P}$. Brinkmann [B], the conjecture is true when $\varphi$ is an automorphism.

\section{EXAmples AND J. HubBard's twisted RABBit PROBlem}

Every quadratic polynomial is affinely conjugate to a unique map of the form $f_{c}(z)=z^{2}+c, c \in \mathbb{C}$. The parameters for which the unique finite critical point at the origin is periodic of period three are $c \approx-1.755$ and $c \approx-.123 \pm .745 i$. Among these, the unique map $f(z)$ for which $\operatorname{Im}(c)>0$ is known as the Douady rabbit; its Julia set is indicated in Figure 1. The critical point is the unique center of symmetry.

Put $A_{0}=P_{f}=\{\infty, 0, c, f(c)\}$ and $A_{1}=f^{-1} P_{f}=A_{0} \cup\{-c,-f(c)\}$. Choose generators for $G_{0}, G_{1}$ as follows.

In the figure, the basepoint $b$ is a fixed point of $f$ and is the prominent black square. Each of the generators $x_{1}, x_{2}, x_{3}$ runs from the basepoint, out the spoke, around the loop as indicated, and back down the spoke to the basepoint. The generators $x_{4}, x_{5}$, in light gray, run from the basepoint down the spoke towards the critical point, loop southwest of the critical point, proceed along the spoke to the 
negative of the fixed point, out the spoke, around the loop as indicated, and then back the way it came.

Combinatorially, the map $f$ restricted to the indicated curves first folds about the critical point onto the top half, then rotates by $1 / 3$ of a turn counterclockwise. From this description it is easy to verify that the map $\varphi_{f}$ is given by sending

$$
\left(x_{1}, x_{2}, x_{3}, x_{4}, x_{5}\right) \mapsto\left(x_{2}^{2}, x_{3}, x_{1}, x_{2} x_{3} x_{2}^{-1}, x_{2} x_{1} x_{2}^{-1}\right)
$$

and is in fact an immersion. Note that $\bigcap_{n>0} \varphi_{f}^{\circ n}\left(G_{1}\right)=\{1\}$ as an application of $\varphi_{f}^{\circ 3}$ doubles the word length of elements of $\left\langle x_{1}, x_{2}, x_{3}\right\rangle$.

Let $\tau:\left(\widehat{\mathbb{C}}, A_{0}\right) \rightarrow\left(\widehat{\mathbb{C}}, A_{0}\right)$ be a right Dehn twist about some curve (such as the dashed curve indicated in the figure) and let $f_{m}=\tau^{m} \circ f, m \in \mathbb{Z}$. The equivalence class of $f_{m}$ depends only on the isotopy class $[\tau]$, which we regard as an element of the mapping class group of the four-times punctured sphere. Any Thurston map of degree two, with a unique fixed critical point (say at infinity), and another critical point of least period three, is equivalent to exactly one of the three polynomials listed above. To see this, observe that a preimage under such a map of a disk in the plane whose boundary is contained in $X_{0}$ is either a disk mapping by degree two branched over a unique point in the interior, or a pair of disjoint disks mapping by degree one, at most one of which contains a periodic point in $A_{0}$. It follows easily that there are no Levy cycles, hence such a map is unobstructed. For a more general statement, see [Lev]. Thus in particular $f_{m}$ is equivalent to exactly one of the above three maps $f_{c}$. J. Hubbard (personal communication) has posed the following problem: determine $f_{c}$ as a function of $m$. A subtlety is that the action of the integers on the set of functions $f_{m}$ given by $n . f_{m}=f_{n+m}$ does not a priori descend to an action of the integers on the set of three equivalence classes, essentially for the same reason that left-multiplication in a group does not in general descend to an action on conjugacy classes.

The map $\tau_{*}: G_{0} \rightarrow G_{0}$ on fundamental groups induced by the Dehn twist $\tau$ about the curve in the figure is given by

$$
\left(x_{1}, x_{2}, x_{3}\right) \mapsto\left(x_{1}, x_{2} x_{3} x_{2} x_{3}^{-1} x_{2}^{-1}, x_{2} x_{3} x_{2}^{-1}\right)
$$

hence e.g. $\varphi_{f_{1}}$ sends

$$
\begin{aligned}
x_{1} & \mapsto x_{2} x_{3}\left(x_{2}^{2}\right) x_{3}^{-1} x_{2}^{-1} \\
x_{2} & \mapsto x_{2} x_{3} x_{2}^{-1} \\
x_{3} & \mapsto x_{1} \\
x_{4} & \mapsto x_{2} x_{3}\left(x_{2} x_{3} x_{2}^{-1}\right) x_{3}^{-1} x_{2}^{-1} \\
x_{5} & \mapsto x_{2} x_{3} x_{2} x_{3}^{-1} x_{2}^{-1}\left(x_{1}\right) x_{2} x_{3} x_{2}^{-1} x_{3}^{-1} x_{2}^{-1}
\end{aligned}
$$

Unfortunately, the maps $\varphi_{f_{m}}$ need not be immersions. For example, under $\varphi_{f_{1}}$, the image of $x_{1}$ begins with $x_{2}$ and ends with $x_{2}^{-1}$. Hence $\varphi_{f_{1}}\left(x_{1}\right) \varphi_{f_{1}}\left(x_{1}\right)$ contains the subword $x_{2}^{-1} x_{2}$, so is not freely reduced. The computation of conjugacy invariants (such as the asymptotic growth rate of word lengths) will likely require computation of the "normal form" via Stallings folds. While perhaps directly tractable for $m=$ \pm 1 , resolution of Hubbard's problem via a direct application of this technology seems unlikely, and other ideas will be needed. 


\section{NOTE ADDED IN PROOF}

A. Kameyama (personal communication) has informed me that the proof of Theorem 5.5 in Kam gives a recursive algorithm for solving Hubbard's problem.

\section{REFERENCES}

[B] P. Brinkmann. Hyperbolic automorphisms of free groups. Geom. Funct. Anal. 10(2000), no. 5, 1071-1089. MR 2001m:20061

[DV] Warren Dicks and Enric Ventura. The group fixed by a family of injective endomorphisms of a free group. American Mathematical Society, Providence, RI, 1996. MR 97h:20030

[DH] A. Douady and John Hubbard. A proof of Thurston's topological characterization of rational functions. Acta. Math. 171(1993), 263-297. MR 94j:58143

[FH] Michael Handel and Mark Feighn. Mapping tori of free group automorphisms are coherent. Ann. of Math. (2) 149(1999), no. 3, 1061-1077. MR 2000i:20050

[GMSW] Ross Geoghegan, Michael L. Mihalik, Mark Sapir, and Daniel T. Wise. Ascending HNN extensions of finitely generated free groups are Hopfian. Bull. London Math. Soc. 33(2001), no. 3, 292-298. MR 2002a:20029

[Kap] Ilya Kapovich. Mapping tori of endomorphisms of free groups. Comm. Algebra 28(2000), 2895-2917. MR 2001c:20098

[Kam] Atsushi Kameyama. The Thurston equivalence for postcritically finite branched coverings. Osaka J. Math. 38(2001), 565-610. MR 2002h:57004

[Lev] Silvio Levy. Critically Finite Rational Maps. Ph.D. thesis, Princeton University, 1986.

[Pil1] Kevin M. Pilgrim. Dessins d'enfants and Hubbard trees. Ann. Sci. École Norm. Sup. (4) 33(2000), 671-693. MR 2002m:37062

[Pil2] Kevin M. Pilgrim. Canonical Thurston obstructions. Adv. Math. 158(2001), 154-168. MR 2001m:57004]

[ST] Mitsuhiro Shishikura and Tan Lei. A family of cubic rational maps and matings of cubic polynomials. Experiment. Math. 9(2000), 29-53. MR 2001c:37042

[Sta] John R. Stallings. Topology of finite graphs Invent. Math. 71(1983), 551-565. MR 85m:05037a

[Tan] Tan Lei. Matings of quadratic polynomials. Ergodic Theory Dynamical Systems 12(1992), 589-620. MR 93h:58129

[ZVC] Heiner Zieschang, Elmar Vogt, and Hans-Dieter Coldewey. Surfaces and planar discontinuous groups. Springer, Berlin, 1980. Translated from the German by John Stillwell. MR 82h:57002

Department of Mathematics, Indiana University, Bloomington, Indiana 47405-7106

E-mail address: pilgrim@indiana.edu 